\title{
Towards rapid nanoscale chemical analysis using tip-enhanced Raman spectroscopy with Ag- coated dielectric tips
}

\author{
Journal Article \\ Author(s): \\ Yeo, Boon-Siang; Schmid, Thomas; Zhang, Weihua; Zenobi, Renato \\ Publication date: \\ 2007 \\ Permanent link: \\ https://doi.org/10.3929/ethz-b-000004025
}

Rights / license:

In Copyright - Non-Commercial Use Permitted

Originally published in:

Analytical and Bioanalytical Chemistry 387(8), https://doi.org/10.1007/s00216-007-1165-7 


\title{
Towards rapid nanoscale chemical analysis using tip-enhanced Raman spectroscopy with Ag-coated dielectric tips
}

\author{
Boon-Siang Yeo • Thomas Schmid • Weihua Zhang • \\ Renato Zenobi
}

Received: 13 December 2006 /Revised: 25 January 2007 / Accepted: 30 January 2007 / Published online: 28 February 2007

(C) Springer-Verlag 2007

\begin{abstract}
The influence of dielectric substrates on the Raman scattering activities of $\mathrm{Ag}$ overlayers has been investigated. Materials with low refractive indices, such as $\mathrm{SiO}_{2}, \mathrm{SiO}_{\mathrm{x}}$ and $\mathrm{AlF}_{3}$, were found to provide suitable supporting platforms for Ag films to give strong surfaceenhanced Raman scattering for dye molecules when illuminated at $488 \mathrm{~nm}$. This finding was then extended to tip-enhanced Raman scattering (TERS). Huge enhancements of 70-80×, corresponding to net enhancements of $>10^{4}$, were observed for brilliant cresyl blue test analyte when Ag-coated tips made from or precoated with low refractive index materials were applied. The yield of fabricated tips that significantly enhance the Raman signals was found to be close to $100 \%$. These findings provide crucial steps towards the use of TERS as a robust technique for rapid chemical imaging with nanometer spatial resolution.
\end{abstract}

Keywords Nanoscale chemical analysis · Surface-enhanced Raman spectroscopy · Tip-enhanced Raman spectroscopy .

Refractive index $\cdot$ Surface plasmon resonance

\section{Introduction}

Tip-enhanced Raman spectroscopy (TERS) has shown great promise as a tool for in situ nanoscale chemical analysis $[1,2]$. The ability to perform such nondestructive measurements is of increasing importance in the rapidly developing areas of nanoscience and nanotechnology. The

B.-S. Yeo $\cdot$ T. Schmid $\cdot$ W. Zhang $\cdot$ R. Zenobi $(\bowtie)$

Department of Chemistry and Applied Biosciences, ETH Zurich, 8093 Zurich, Switzerland

e-mail: zenobi@org.chem.ethz.ch capability to "see" chemical species at surfaces with a spatial resolution below the optical diffraction limit is, for example, necessary to gain a proper understanding of the mechanisms involved in commercially valuable catalytic processes. In the fabrication and quality control of miniaturized organic-based optical, electroluminescent devices and sensors, the ability to image the nanosize morphology and defects of ultrathin molecular films is indispensable [3].

TERS is essentially a hybrid system that harvests and combines the nanometer resolution afforded by scanning probe microscopy (SPM) with the molecular fingerprinting available from surface-enhanced Raman spectroscopy (SERS). One effective TERS set-up employs an atomic force microscope (AFM) in tandem with an inverted microscope, which optimizes both the excitation of the Raman-active tip and the collection of the weak Raman signals $[1,4,5]$. The AFM has an advantage over other SPM systems because of its ability to obtain complimentary data, such as friction force images, simultaneously with the topography. TERS measurements utilizing such set-ups have been performed for dye molecules $[1,5,6]$, carbon allotropes such as single-walled carbon nanotubes [7], dye-sensitized $\mathrm{TiO}_{2}$ nanoparticles [8], DNA components [9], and bacteria samples [10].

At the heart of these TERS experiments is the use of a laser-illuminated metal-coated AFM tip capable of electromagnetically enhancing the intrinsically weak Raman signals through the generation of localized surface plasmons (LSPs). These TERS probes can be fabricated by vapor-coating the AFM tips with $\mathrm{Ag}$ or Au nanoparticles, in a process analogous to the metallization of glass slides used for SERS [5, 11]. Due to their similarities, as illustrated in the gedankenexperiment shown in Fig. 1, physical principles gathered from the extensive body of experience in SERS could therefore be adapted to TERS. 
Fig. 1 A gedankenexperiment where TERS is considered to be an inverted SERS set-up. The roughened Ag metal film is replaced with a metallized AFM tip that is scanned over the analyte. The spatial resolution of TERS is limited by the size of the enhancing Ag nanoparticle

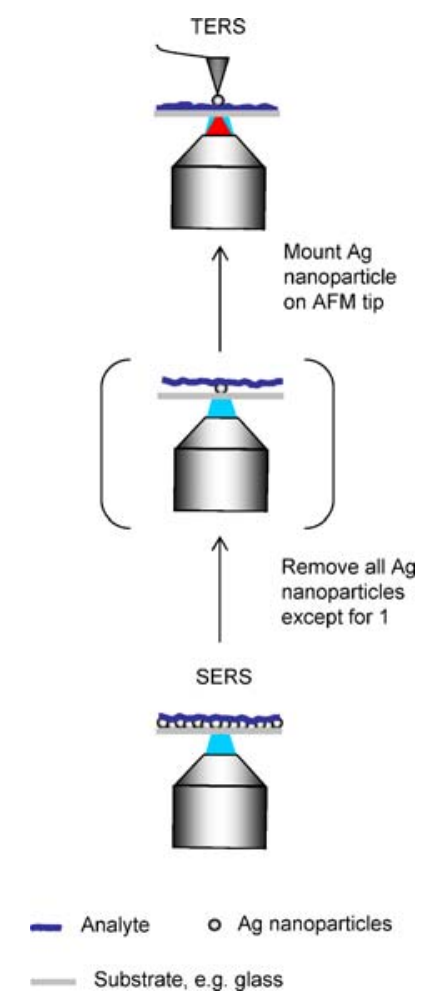

In general, the two enhancement mechanisms accepted for SERS and TERS are electromagnetic and chemical enhancement $[12,13]$. The former depends strongly on the extent of resonance of two wavelengths belonging to the illumination laser and the scattered Raman signal(s) with the wavelength of the surface plasmon resonance $\left(\lambda_{\mathrm{SPR}}\right)$ of the enhancing metal nanoparticles. The use of tunable lasers would be the best way to achieve this condition. However, this may result in experimental difficulties, such as crosstalk with the four-quadrant photodetector of the AFM, and it would also necessitate the integration of more optical components such as costly holographic notch filters into the system. Hence, low-wavelength lasers are normally used in AFM-TERS experiments [1, 6,9]. One such wavelength is $488 \mathrm{~nm}$, which is widely used for fluorescence microscopy. In Raman spectroscopy, due to the "fourth power rule", the intensity of the scattered signal is stronger when a shorter illumination wavelength is employed [14]. This means that excitation with $488 \mathrm{~nm}$ should be better for optimizing signal strength than the use of red wavelengths. Moreover, huge SERS enhancements with low excitation wavelengths such as $488 \mathrm{~nm}$ have been reported for molecules such as Rhodamine 6G (R6G) adsorbed onto Ag colloids [15]. From these considerations, it appears that this wavelength should be ideal for performing effective TERS and fluorescence measurements in the same set-up. However, only low TERS enhancements have been obtained theoretically and experimentally at $488 \mathrm{~nm}[4,6,16,17]$. For instance, when this illumination wavelength was used, the
Raman spectra of R6G and brilliant cresyl blue $(\mathrm{BCB})$ thin films were enhanced by only $\sim 1.5 \times$ in the presence of a $\mathrm{Ag}$ coated Si tip.

One possible key to understanding this shortfall lies in the optical properties of the Ag nanoparticles in relation to the illuminating laser wavelength. The $\lambda_{\mathrm{SPR}}$ of Ag nanoparticles depends on their shapes, sizes, degree of aggregation and their environment [18-22]. It has been shown to redshift with increasing refractive index $(n)$ of the underlying substrate. For instance, the $\lambda_{\mathrm{SPR}}$ of sputtered $\mathrm{Ag}$ island films deposited onto $\mathrm{SiO}_{2}(n=1.5)$ lies in the range of 444-506 nm [21]. When $\mathrm{TiO}_{2}(n=2.75)$ is employed instead, the $\lambda_{\mathrm{SPR}}$ increases to $600 \mathrm{~nm}$. In the context of TERS experiments, this finding implies that the AFM tip material plays a crucial role in supporting the localized surface plasmons (LSPs) of the Ag nanoparticles, thereby influencing the enhancement of the Raman signals. Commercially available AFM tips made of $\mathrm{Si}$ are almost exclusively used in TERS experiments, due to their availability and low cost. Si has a far higher $n$ of 4.4 at $488 \mathrm{~nm}$ illumination. Under its dielectric influence, the $\lambda_{\text {SPR }}$ of the attached metal particles will increase. This leads to the generation of a weaker LSP at $488 \mathrm{~nm}$ excitation. This physical principle has so far, rather surprisingly, been neglected in TERS, which may in part explain the generally poor TERS enhancements reported in the literature [4-6, 17]. Therefore, the yield of fabricated tips that significantly enhance the Raman signal has also been low, typically $20-30 \%$ or even less. These factors are undoubtedly serious barriers that have prevented the application of TERS as an analytical technique.

In this work, several issues are addressed:

- Given the restrictions on the laser wavelengths and substrate materials that can be used, are there any simple methods that would allow us to qualitatively determine the best AFM tips to use for a particular excitation wavelength?

- Is there a way to modify the tips such that they become suitable for TERS at a particular excitation wavelength?

The first question is answered experimentally: the SERS activities of dye molecules on $\mathrm{Ag}$ deposited on $\mathrm{Si}(n=4.4)$, $\mathrm{SiO}_{\mathrm{x}}(n=1.5-2.05), \mathrm{SiO}_{2}(n=1.5)$ and $\mathrm{AlF}_{3}(n=1.4)$ substrates were studied at $488 \mathrm{~nm}$ excitation. It was found that the enhancement of the Raman signals of the analytes increases with decreasing $n$ of the underlying dielectric platform. This result was then extended to TERS with Agcoated $\mathrm{SiO}_{2}$ tips, as well as commercial $\mathrm{SiN}$ and $\mathrm{Si}$ tips precoated with $\mathrm{SiO}_{x}$ and $\mathrm{AlF}_{3}$. Good enhancements of $\mathrm{BCB}$ thin-film analyte were obtained using these modified tips, as well as a high yield of "hot" tips in the fabrication process. The findings in this work mark a crucial step 
towards the use of TERS as a robust technique for nanoscale chemical analysis.

\section{Experimental}

Instrumentation The set-up used in this work has been described previously [4]. Briefly, it consists of an inverted confocal microscope (FV500, IX70, Olympus, Tokyo, Japan), an atomic force microscope (Explorer, Veeco, Santa Barbara, CA, USA) and a Raman spectrograph (Holospec, Kaiser Optical Systems, Inc., Ann Arbor, MI, USA). A $488 \mathrm{~nm}$ laser (ChromaLASE, Blue Sky Research, Milpitas, CA, USA) was used as the excitation source.

Chemicals and sample preparation For the SERS experiments, Si substrates were cut from wafers (B-doped, (100), Siliconquest Int., Santa Clara, CA, USA) and cleaned in toluene and acetone. Glass slides $(\mathrm{n} \approx 1.5$; Paul Marienfeld $\mathrm{GmbH} \&$ Co. KG, Lauda-Königshofen, Germany) were cleaned in piranha solution (concentrated $\mathrm{H}_{2} \mathrm{SO}_{4}: \mathrm{H}_{2} \mathrm{O}_{2}$, 3:1) for ten minutes. $\mathrm{SiO}_{2}$ tips were supplied by the Institute of Microelectronics, University of Neuchâtel, Switzerland. Commercially available contact mode AFM tips made of Si (PPP-CONTR, NanoSensors, Neuchâtel, Switzerland) and SiN (RC800PSA, Olympus) were also employed in this work. The samples were mounted in a vapor-coating chamber (MED 020, Bal-Tec, Balzers, Lichtenstein) $15 \mathrm{~cm}$ directly above the evaporation source. Twenty nanometer $\mathrm{SiO}_{\mathrm{x}}$ (Sigma-Aldrich, Buchs, Switzerland; 99.99\%) or $\mathrm{AlF}_{3}$ (Sigma-Aldrich, 99\%) were deposited on some of the samples from Mo-baffled boxes (to prevent "spitting") at rates of $0.1 \mathrm{~nm} / \mathrm{s}$. Ag (Bal-Tec, 99.99\%) was subsequently coated onto the dielectric surfaces at a rate of $0.05 \mathrm{~nm} / \mathrm{s}$. The pressure of the system did not rise above $5 \times 10^{-5}$ mbar during the coating process. The $n$ of pure $\mathrm{SiO}$ films is 2.05 at $488 \mathrm{~nm}$ [23]. However, $\mathrm{SiO}$ is known to getter ambient oxygen gas during the deposition film process, leading to a lowering of $n$. Hence, these films will henceforth be termed " $\mathrm{SiO}_{\mathrm{x}}$ " films.

The analyte molecules used were BCB (Fluka, Buchs, Switzerland), R6G (Sigma-Aldrich) and malachite green, (MG, Fluka). They were dissolved in methanol, and $5 \mu \mathrm{L}$ were spin-coated onto the Ag surfaces. For the TERS experiments, $\mathrm{BCB}$ was spin-coated directly onto clean glass surfaces to give transparent bluish thin films.

SERS experiments The SERS experiments utilized a $0.7 \mathrm{~N}$. A., $20 \times$ objective focusing $20 \mu \mathrm{W}$ light from a $488 \mathrm{~nm}$ laser onto the samples. Scattered light was collected through the same objective and fed into the Raman spectrograph through the fiber optics. Spectra were collected from at least ten nonoverlapping spots scattered throughout a sample and averaged.

TERS experiments $2-5 \mu \mathrm{W}$ light from a $488 \mathrm{~nm}$ laser was focused onto the sample and an Ag-coated tip using a 1.4 N.A., $60 \times$ oil-immersion objective.

No background subtraction or smoothening of the spectra was made. The data points were processed using Igor Pro Version 4.09A Carbon (WaveMetrics, Inc., Lake Oswego, OR, USA).

\section{Results}

\section{TERS enhancement factors}

In this work, the observed enhancement (OE) is defined as the ratio of the intensities of a chosen band in the Raman spectrum with and without the presence of the tip. The net enhancement (NE) takes into account the facts that the sources of the near-field and far-field signals are different and that the intensities are normalized to the corresponding illuminated areas or volumes. The NE can be calculated using Eq. 1 [5]:

$N E=\frac{(O E-1) \cdot r_{\text {far-field }}^{2}}{r_{\text {near }- \text { field }}^{2}}$

where $r$ is the radius of the illuminated area.

The OE will directly determine the recording speed and quality of the TERS images. Since it is of greater practical relevance, it will be used to evaluate the TERS spectra presented in this work.

\section{SERS of dye molecules on Ag/dielectric surfaces}

The sets of SER spectra of BCB, MG and R6G spin-coated onto $6 \mathrm{~nm}$ of $\mathrm{Ag}$ vapor coated onto $\mathrm{Si}, \mathrm{SiO}_{\mathrm{x}}, \mathrm{SiO}_{2}$ and $\mathrm{AlF}_{3}$ surfaces are shown in Fig. 2. Their Raman intensities as a function of the $n$ of the underlying substrate are also plotted in Fig. 3. The vibrational signatures of the SER spectra are consistent with the Raman spectra of the bulk species and those deposited on noble metal substrates [4, 11, 24]. For a given sample, the SERS intensities observed at different locations are usually the same (maximum observed deviation $<20 \%$ ). It can be observed that the Raman signals exhibited by the dye molecules are strongest on the $\mathrm{Ag}$ / $\mathrm{AlF}_{3}$ system and weakest on $\mathrm{Ag} / \mathrm{Si}$, with the difference in signal strength reaching at least one order of magnitude. The Raman enhancements of the molecules on $\mathrm{Ag} / \mathrm{SiO}_{\mathrm{x}}$ are close to those of the $\mathrm{Ag} / \mathrm{SiO}_{2}$ substrates. This can be attributed to the stoichiometry of the $\mathrm{SiO}_{\mathrm{x}}$ layer resembling $\mathrm{SiO}_{2}$. SER spectra of the same intensity have also been 


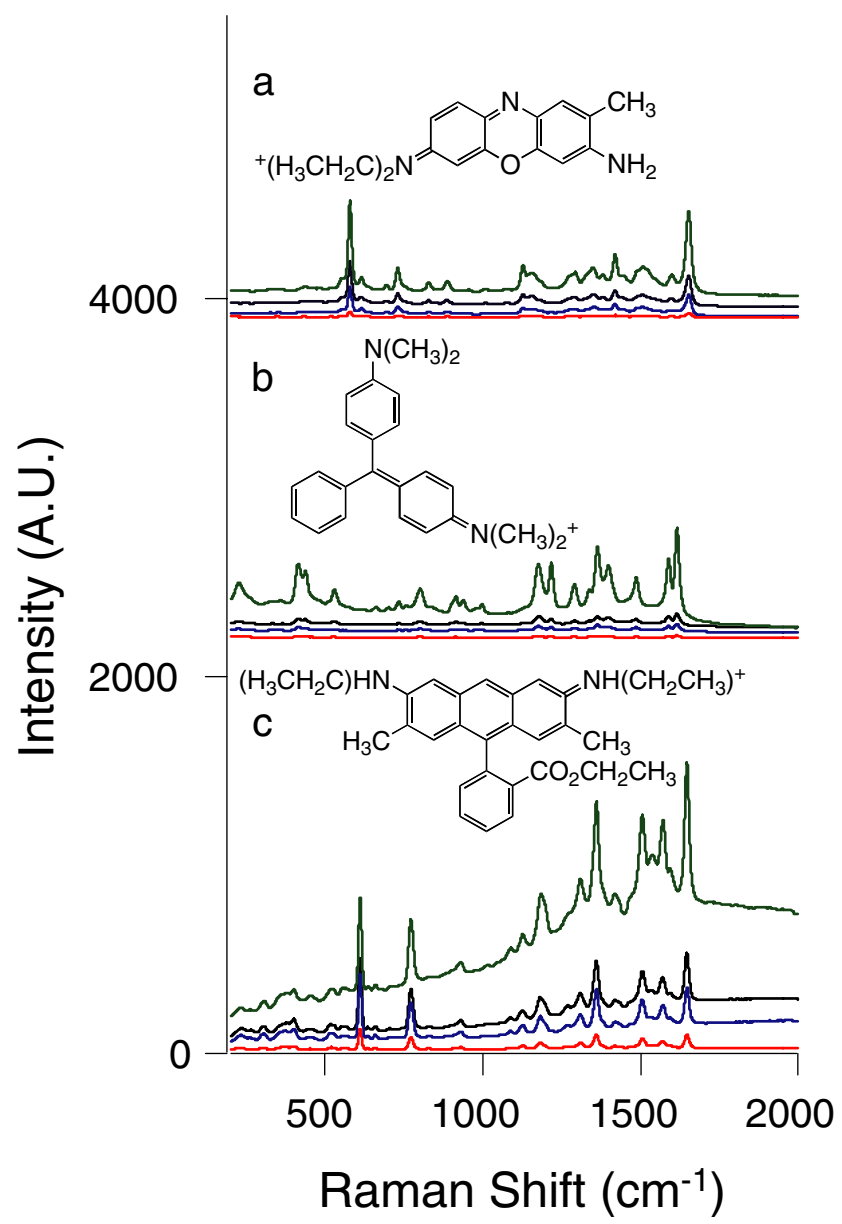

Fig. 2a-c Averaged SERS spectra of dye molecules spin-coated onto $6 \mathrm{~nm}$ of $\mathrm{Ag}$ deposited onto $20 \mathrm{~nm}$ of $\mathrm{AlF}_{3}$ (green trace), $\mathrm{SiO}_{2}$ (black), $\mathrm{SiO}_{\mathrm{x}}$ (blue) or $\mathrm{Si}($ red $)$ : a $\mathrm{BCB}, \mathbf{b} \mathrm{MG}$ and $\mathbf{c}$ R6G. The intensities of the Raman signals are shown to increase as a function of decreasing refractive index of the underlying substrates. The chemical structures of the dye molecules are included above the spectra. The collection time for each spectrum was $1 \mathrm{~s}$

experimentally obtained from substrates fabricated from $20 \mathrm{~nm} \mathrm{SiO}_{\mathrm{x}}$ deposited on either $\mathrm{Si}$ or $\mathrm{SiO}_{2}$ (data not shown). This demonstrates that the deposited overlayer can mask the dielectric influence of the substrates.

The difference in intensities of the SER spectra as a function of $n$ of the underlying dielectric substrate can be attributed to the $\lambda_{\mathrm{SPR}}$ of $\mathrm{Ag}$ being blueshifted when it is deposited on $\mathrm{AlF}_{3}$ (or $\mathrm{SiO}_{\mathrm{x}}, \mathrm{SiO}_{2}$ ) as compared to $\mathrm{Si}$. This results in a larger field enhancement, since the $\lambda_{\text {SPR }}$ would be in better resonance with $488 \mathrm{~nm}$. This finding is in agreement with previous findings that the $\lambda_{\mathrm{SPR}}$ of $\mathrm{Ag}$ island films deposited on $\mathrm{SiO}_{2}$ lies in the range 444-506 nm (a narrow range of $52 \mathrm{~nm}$ ) [21]. With increasing $n$ of the substrate, the $\lambda_{\text {SPR }}$ was found to redshift.

TERS using commercially available modified tips

From a physical point of view, TERS performed with the present set-up is effectively the same as a conventional

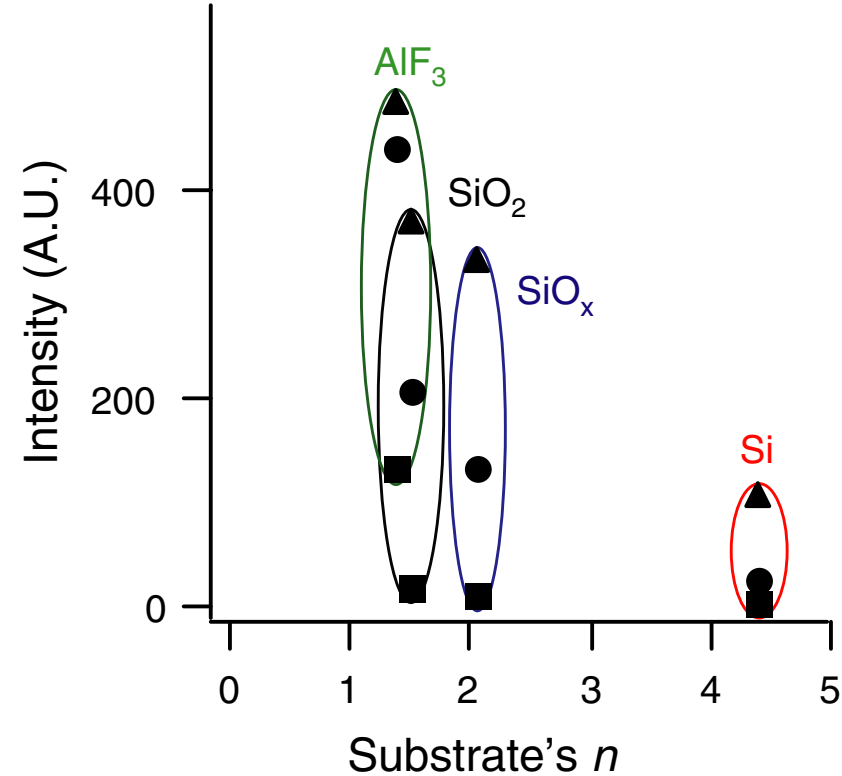

Fig. 3 Plot of integrated SERS intensities as a function of refractive index of the underlying surfaces. Circles, BCB; squares, MG; triangles, $\mathrm{R} 6 \mathrm{G}$

SERS experiment with a roughened metal substrate, although a superior spatial resolution is achieved. As such, the intensities of the SERS signals for molecules on $\mathrm{Ag}$ island films deposited on $\mathrm{Si}, \mathrm{SiO}_{\mathrm{x}}, \mathrm{SiO}_{2}$ and $\mathrm{AlF}_{3}$ are expected to shed some light on which would be the best choice of AFM tip substrate to use with $488 \mathrm{~nm}$ illumination. The present results suggest that a judicious choice of AFM tip substrate is crucial to maximizing the Raman enhancement. From the intensity variations exhibited, Si substrates are clearly the most unsuited for SERS using $488 \mathrm{~nm}$, while $\mathrm{SiO}_{\mathrm{x}}, \mathrm{SiO}_{2}$ and $\mathrm{AlF}_{3}$ are better. These results are now extended to TERS measurements.

$\mathrm{BCB}$ is selected as the analyte because it does not fluoresce with $488 \mathrm{~nm}$ excitation. Thin films are used as test systems because they are currently attracting much attention due to their potential uses in molecular-based electronic devices. Figure 4 shows the TER spectra of BCB thin films enhanced with Ag-coated dielectric tips. For comparison, Raman and SER spectra of bulk BCB are also presented in Fig. 4a and $\mathrm{b}$, respectively. The vibrational signatures of $\mathrm{BCB}$ in the TER spectra (black traces) in Fig. $4 \mathrm{c}-\mathrm{e}$ agree well with the frequencies observed in the bulk Raman spectra of $\mathrm{BCB}$ and those shown in the literature, with the two most prominent bands at 584 and $1662 \mathrm{~cm}^{-1}$ [4]. With an $\mathrm{Ag}$-coated $\mathrm{SiO}_{2}$ tip, an $\mathrm{OE}$ of 70 $80 \times$ is attained, as shown in Fig. 4c.

A method that can be used to modify commercially available SiN and Si AFM tips for TERS measurements is now presented. These are first vapor-coated with $20 \mathrm{~nm}$ $\mathrm{AlF}_{3}$ or $\mathrm{SiO}_{\mathrm{x}}$ to modify the surface dielectric environment of the substrate, followed by $20-30 \mathrm{~nm} \mathrm{Ag}$. Typical 


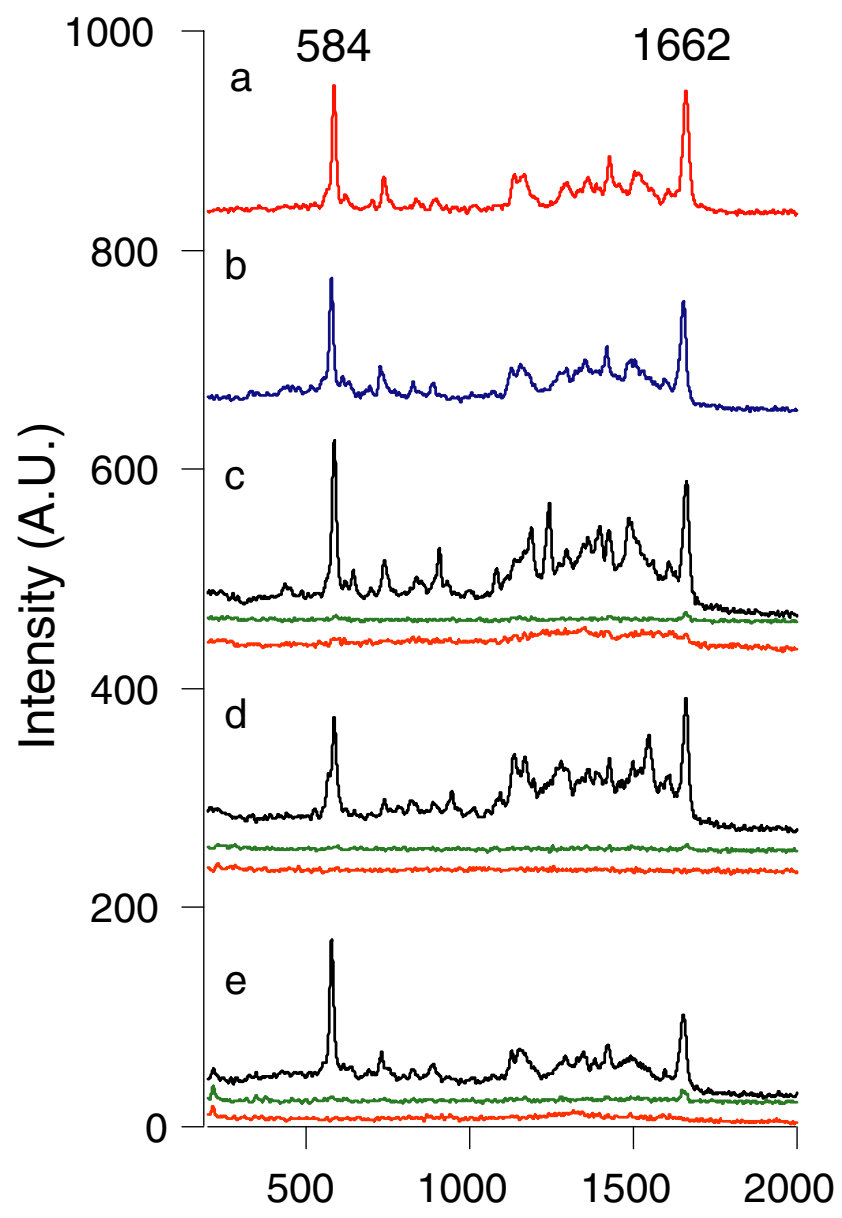

Raman Shift $\left(\mathrm{cm}^{-1}\right)$

Fig. 4a-e a Raman spectrum of BCB, b SERS of BCB spin-coated onto Ag film. The Raman spectrum of $\mathrm{BCB}$ acquired with tip in contact (black) and retracted (green trace) are shown for Ag-coated $\mathrm{SiO}_{2}(\mathbf{c}), \mathrm{SiO}_{\mathrm{x}} / \mathrm{SiN}(\mathbf{d})$, and $\mathrm{AlF}_{3} / \mathrm{SiN}$ (e) tips. The orange traces are the tips' spectra collected after the experiments. The collection time for each spectrum was $10 \mathrm{~s}$

scanning electron microscopy (SEM) images of these tips are shown in Fig. 5. The appearances of the tips are similar to those reported for Ag metal deposited directly onto $\mathrm{SiN}$ tips with average apex diameters of $40 \mathrm{~nm}$ [5]. This demonstrates that the precoated underlying dielectric layer does not drastically affect the morphology of the $\mathrm{Ag}$ coatings.

The TER spectra of a BCB thin film obtained using these tips are shown in Fig. 4d and e, where OEs of 70-80× were achieved. The NEs obtained in this work can be estimated to be $>10^{4}$ with Eq. 1, using values of 30 and $700 \mathrm{~nm}$ for the diameters of the metal particle at the tip apex and the laser focus area, respectively. It should be emphasized here that this value is a lower limit since earlier works have demonstrated that the actual near-field illuminated area is smaller than the size of the tip apex $[5,7]$.
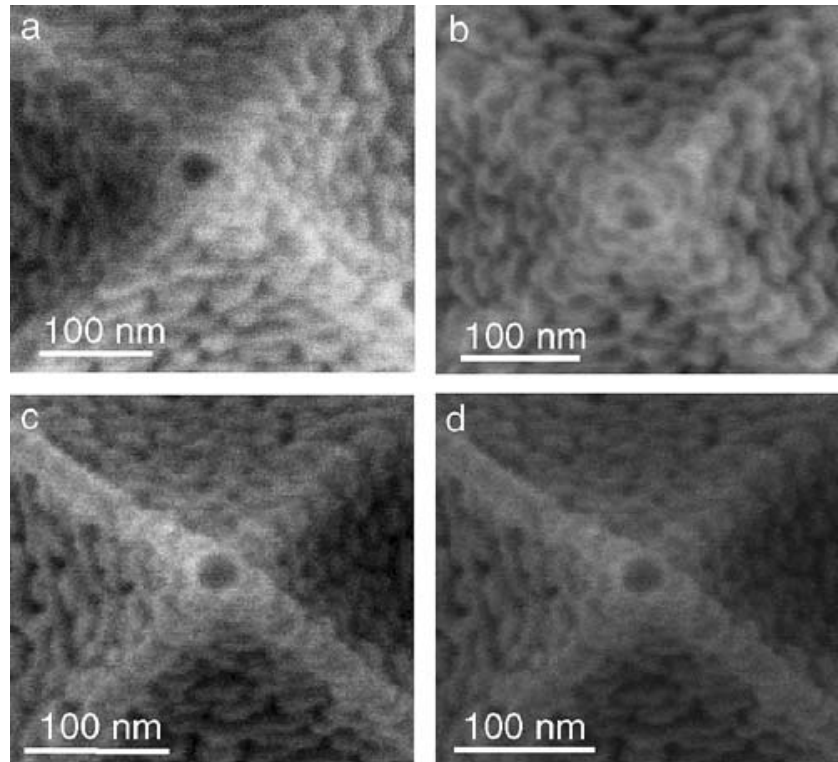

Fig. 5a-d SEM images of the dielectric-precoated tips vapor-coated with Ag nanoparticles. The tip apex is located in the middle of each image. Tips pre-coated with $\mathrm{SiO}_{\mathrm{x}}$ are shown in $\mathbf{a}$ and $\mathbf{b}$, while those with $\mathrm{AlF}_{3}$ are in $\mathbf{c}$ and $\mathbf{d}$

More importantly, the OEs presented here surpass all other previous studies with similar set-ups, including those utilizing BCB thin films with $\mathrm{Ag}$-coated $\mathrm{SiN}(n=2.05)$ or Si tips $[1,4,5,25]$. The attainment of high OEs is a crucial step towards the use of TERS as a standard tool for rapid nanoscale chemical imaging. Furthermore, the percentage of $\mathrm{Ag} / \mathrm{AlF}_{3}$ tips exhibiting reasonable TERS enhancement is close to $100 \%$. These tips have also been successfully used in our laboratory for TERS studies on $\mathrm{MG}, \mathrm{C}_{60}$, and dipicolinic acid, a bacteria marker.

It can be observed in Fig. $4 \mathrm{e}$ that the band at $584 \mathrm{~cm}^{-1}$ $(502 \mathrm{~nm})$ is more enhanced than the one at $1662 \mathrm{~cm}^{-1}$ $(531 \mathrm{~nm})$. This can be attributed to a larger resonance enhancement by the LSP of the tip apex's Ag particles at $502 \mathrm{~nm}\left(584 \mathrm{~cm}^{-1}\right.$ band), which also demonstrates the proximity to $488 \mathrm{~nm}$ and the relatively narrow bandwidth of the $\lambda_{\mathrm{SPR}}$ of the Ag particles supported on $\mathrm{AlF}_{3}$. These Raman intensity ratios could potentially be used as simple ways to determine the location and shape of the LSP band of the TERS tips.

Several TER signals in the $1100-1600 \mathrm{~cm}^{-1}$ range, which are not consistent with those of the bulk BCB, are also present (Fig. 4c and d). These peaks could possibly arise from the enhancement of contaminations (e.g., carbon) present in the $\mathrm{BCB}$ layer or on the tip, since the experiments were performed in an ambient environment. After the TERS measurements, the Raman spectra (orange traces) of the tips themselves were collected. Small quantities of carbon could be identified on the $\mathrm{Ag} / \mathrm{SiO}_{2}$ tip shown in Fig. 4c. No BCB molecules were detected on any 
of the tips (Fig. 4c-e). This demonstrates that the enhancement mechanism of the Raman signals is electromagnetic in nature. Possible methods of reducing and eliminating tip contamination include rinsing with methanol, ozone treatment of the tip, or chemical displacement using thiols $[26,27]$.

To further verify the importance of the surface plasmon resonance of the metal particles in the Raman signal enhancement, TERS measurements with $\mathrm{AlF}_{3} / \mathrm{SiN}$ tips that were similarly coated, albeit with $20 \mathrm{~nm}$ Au instead of Ag, were made. Isolated $\mathrm{Au}$ nanoparticles are known to exhibit their LSP in the green or higher wavelengths, and are unlikely to be suitable for TERS with $488 \mathrm{~nm}$ excitation even when supported on AFM tips with low $n$ [28]. As expected, little enhancement could be obtained from the TERS experiments (data not shown).

An application of TERS: investigation of the chemical composition of a molecular thin film

A powerful application of TERS is its use in studying the morphology and chemical composition of thin films down to nanometer resolution. Figure 6a shows a sequence of TER spectra for a BCB thin film spin-coated on a glass slide. The sample was scanned at $50 \mathrm{~nm}$ intervals along a straight line, with the tip held stationary. Only minor intensity variations were observed, indicating that the thin film was only slightly inhomogeneous at the nanometer level. A larger line scan with TERS data acquired at regular intervals over $4000 \mathrm{~nm}$ was also recorded and is shown in Fig. 6b. At positions 1, 5, 6 and 10, the spectra collected showed no trace of BCB signals, indicating that the film is actually inhomogeneous on this coarser scale. This can be attributed to the $\mathrm{BCB}$ molecules segregating into sub- $\mu \mathrm{m}$-sized islands instead of spreading out evenly during the spin-coating process.

The spectra collected are free from foreign vibrational bands, thereby demonstrating the purity of the sample. The appearance of Raman signals at these irregular intervals also serves as a demonstration of the cleanliness of the tips. This is an important consideration, especially when performing mapping experiments with unknown samples. The presence of foreign molecules on the tip will result in the observation of persistent Raman signals, which would give an inaccurate reflection of the true nature of the sample.

\section{Discussion}

Strategy of the experiment

The purpose of this work was to identify parameters suitable for the fabrication of TERS tips capable of strongly enhancing Raman signals. For efficient TERS or SERS, the
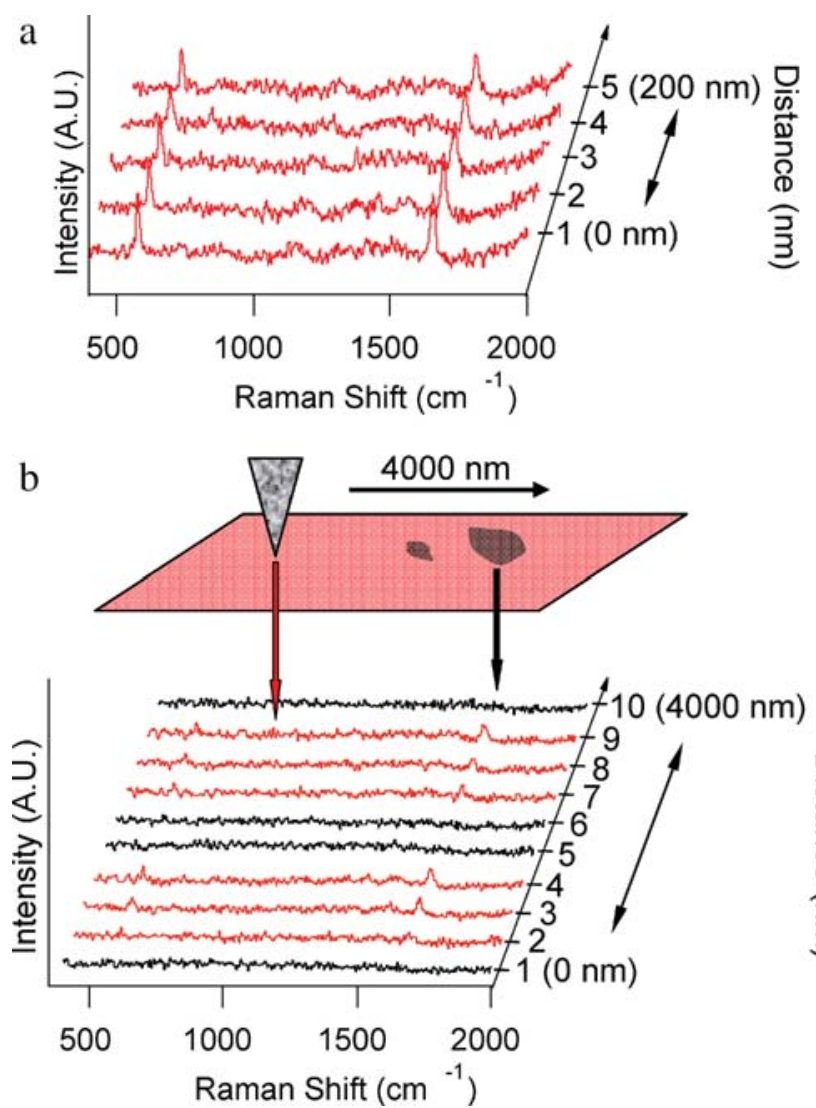

Fig. 6a, b TERS line scan performed at equal intervals of a very dilute BCB thin film along $200 \mathrm{~nm}$ (a) and $4000 \mathrm{~nm}$ (b) lines. The spectra collected at positions 1, 5, 6 and 10 (black traces) are free from vibrational features, which indicate a local absence of BCB. The intensities of the peaks in Fig. $6 a$ and $b$ are different due to the different enhancements exhibited by the two Ag-coated tips. The change in spectral features is attributed to the local absence of $\mathrm{BCB}$ at the enhancement zone of the tip during sample scanning (see cartoon above the spectra). No Raman signals are observed in the absence of the tips in the laser focus area. The collection time for each spectrum was $5 \mathrm{~s}$

wavelength and height of the resonance are important. A qualitative description of the methodology of the experiment is presented here. The optical properties of Ag films can be described by the Maxwell-Garnett theory shown in the following equation:

$\lambda_{\mathrm{SPR}}=\lambda_{\mathrm{p}}\left[\alpha\left(\frac{n^{2}+1}{2}\right)+\varepsilon_{\infty}\right]^{1 / 2}$

where $\lambda_{\mathrm{p}}$ is the plasma wavelength of the bulk $\mathrm{Ag}, \alpha$ is a parameter describing the degree of aggregation and the shape of the metal nanoparticles, and $\varepsilon_{\infty}=$ is the highfrequency dielectric constant due to interband transitions of electrons [21].

Maximum electromagnetic enhancement leading to strong SER scattering occurs when the illumination wavelength is in resonance with the $\lambda_{\mathrm{SPR}}$, as expressed in (2). Equation 2 demonstrates that the $\lambda_{\mathrm{SPR}}$ of the $\mathrm{Ag}$ nanoparticles is 
dependent on the $n$ of the underlying substrate, i.e., $\lambda_{\text {SPR }}$ increases as a function of $n$. From numerous experimental studies, the extinction spectra of $\mathrm{Ag}$ films deposited on $\mathrm{SiO}_{2}$ have been shown to occur at blue wavelengths [21, 22, 29]. When a substrate with a higher $n$ is used, the absorption profile redshifts considerably (by hundreds of $\mathrm{nm}$ ). In accordance with these investigations, the strategy employed in this work is to use substrate materials with low $n$ as SERS and TERS platforms with $488 \mathrm{~nm}$ excitation.

\section{Choice of coating materials}

The choice of coating materials suitable for modifying the surfaces, especially those used for the AFM tips, hinges on several criteria. The material must have a low $n$, be robust, and be easily evaporated. $\mathrm{AlF}_{3}$ fulfils these requirements; it has an $n$ of 1.4 at $488 \mathrm{~nm}$ [30]. Having a well-defined stoichiometry implies that its refractive index should be easily predictable. $\mathrm{AlF}_{3}$ also has a low sublimation temperature $\left(1100{ }^{\circ} \mathrm{C}\right)$ and can easily be evaporated by resistive heating. Other suitable materials identified from this work are $\mathrm{SiO}_{2}$ and $\mathrm{SiO}_{\mathrm{x}}$. $\mathrm{SiO}_{\mathrm{x}}$ has a drawback related to its preparation, because the vapor deposition conditions (such as rate and ambient pressure) play a decisive role in determining the value of $\mathrm{x}$, which can vary from 2 to 1 . This will result in films with $n$ values ranging from 2.05 to 1.5. These silicon oxides also have higher evaporation temperatures. Depositions of $\mathrm{SiO}_{2}$ by e-beam methods have the disadvantage of sometimes resulting in the formation of impure films, due to decomposition at high evaporation temperatures [31].

\section{Insights from SERS experiments as related to TERS}

Performing SERS with different dielectric substrates demonstrates the importance of the underlying support to the enhancement. The SERS intensity has been experimentally shown to increase when the $n$ of the underlying substrate decreases. Such an effect is usually neglected in SERS or TERS studies, with the focus primarily on the shapes and sizes of the enhancing metallic nanoparticle itself. The SERS intensities of dye molecules spin-coated onto a $\mathrm{Ag} / \mathrm{Si}$ surface have been found to be the lowest. This result is consistent with previous TERS results which showed that Ag-coated Si AFM tips do not generally give good Raman enhancement, especially at blue wavelengths such as $488 \mathrm{~nm}[4,6]$. Stronger enhancements were found when longer wavelengths are used (e.g., $785 \mathrm{~nm}$ ) or when the tip material is of low $n$. Data from prior TERS studies have been summarized in [5].

The results from the present SERS study could not strictly eliminate signal contributions caused by "hot spots" not treated adequately by the Maxwell-Garnett theory, or by morphological differences in the Ag surfaces. Their exact physical nature and their relationship to the SERS intensity are not well understood [32]. However, these two effects are not thought to dominate the observed signal trends, since: (a) no isolated spots of particularly high intensity were observed in the confocal images or Raman spectra, and (b), from SEM studies of the SERS substrates, the morphologies of the $\mathrm{Ag} / \mathrm{AlF}_{3}, \mathrm{Ag} / \mathrm{SiO}$ x and $\mathrm{Ag} / \mathrm{SiO}{ }_{2}$ surfaces were found to be very similar.

This work also suggests a simple method of improving the SERS sensitivity of commonly used Ag vapor-coated surfaces for chemical analysis. Roughened $\mathrm{Ag} / \mathrm{AlF}_{3}$ surfaces have advantages over Ag films deposited over polyorganic spheres because $\mathrm{AlF}_{3}$ do not give vibrational bands above $500 \mathrm{~cm}^{-1}$, and there is no possibility of dissociation leading to carbon contamination.

\section{TERS in nanoscale chemical analysis}

In the present work, the TERS bands shown in Fig. 4c-e can be unambiguously assigned to BCB because of the strong intensities of the two bands at 584 and $1662 \mathrm{~cm}^{-1}$. This seemingly trivial point is actually very important because the usefulness of TERS for surface analysis lies in the ability to observe a real vibrational signature of the analyte being investigated. Two TERS investigations of malachite green dyes adsorbed on Au films have recently been reported. Domke et al. presented fingerprint-quality TERS data which was ascribed to scattering from only five dye molecules [33]. On the other hand, the spectra from Neacsu et al. showed great signal fluctuations in intensities and Raman shifts which were attributed to a single molecule moving within the enhancement zone [34]. For the latter study, the presence of carbon contamination from photobleached MG cannot be ruled out, and this constitutes a serious drawback for TERS as a reliable analytical technique.

A TERS mapping of a BCB thin film has also been performed in this work, and the spatial and chemical homogeneity of the film could be determined. A near-field Raman map of a mixture of R6G and crystal violet spincoated onto a rough Ag surface has been previously recorded with a similar set-up [35]. Strong Raman signals due to extra enhancement afforded by the Ag film were observed. However, such investigations suffer from the fact that the tip-molecule-surface configuration varies across the sample. Excitation of an "optical cavity" may occur at certain spots to give stronger TERS signals. This intensity would thus not be indicative of the actual number of dye molecules present, but rather of the local field enhancements. Performing STM-TERS on atomically smooth metal surfaces as supporting platforms may alleviate the problem partially. However, this limits the maximum film thickness to a few monolayers [33]. Moreover, chemisorption of the molecules to the metal atoms may undesirably modify the 
original electronic properties of the film. The present work uses only the Ag tip to amplify the Raman signals of the dye molecules. While the NE is lower than those obtained from gap-mode type experiments $\left(10^{6}\right)$, the more homogeneous Raman enhancement of the sample makes this method more powerful for quantitative chemical analysis.

It should be emphasized that a very low incident laser power of $2-5 \mu \mathrm{W}$ was used throughout the TERS experiments, and the collection time for each TER spectrum is 5 or $10 \mathrm{~s}$. This is in contrast with many previous experimental configurations where light intensities of up to few $\mathrm{mW}$ were used, often with long signal accumulation periods. The high-quality TER spectra presented in this work are due to the effectiveness of the Ag-coated dielectric tips at enhancing the Raman signals and optimization the instrument's detection sensitivity.

\section{Conclusion}

The influence of dielectric substrates on the Raman enhancement of $\mathrm{Ag}$ layers with $488 \mathrm{~nm}$ illumination has been investigated in this work. Those made of or coated with materials with a low refractive index, such as $\mathrm{SiO}_{2}$, $\mathrm{SiO}_{\mathrm{x}}$ and $\mathrm{AlF}_{3}$, have been found to be platforms that yield strong SERS scattering. Si was found to be unsuitable for use as a SERS substrate at $488 \mathrm{~nm}$ illumination. This result is consistent with the low TERS enhancement previously observed with Ag-coated Si tips.

TERS enhancements with OEs of 70-80× were achieved when $\mathrm{BCB}$ was used as test analyte and $\mathrm{Ag}$-coated $\mathrm{SiO}_{2}$ tips were employed. Commercially available $\mathrm{SiN}$ or $\mathrm{Si}$ tips precoated with $\mathrm{SiO}_{\mathrm{x}}$ or $\mathrm{AlF}_{3}$ and later deposited with $\mathrm{Ag}$ gave equally high TERS enhancements. TERS line-scans of a BCB thin film were performed and inhomogeneities were detected at the sub- $\mu \mathrm{m}$ level. The OEs reported in this work surpass those reported for all previous studies with similar setups. A yield of enhancing $\mathrm{Ag} / \mathrm{AlF}_{3}$ TERS tips of close to $100 \%$ was also obtained. The present findings provide important steps towards the use of TERS as a standard technique for rapid nanoscale chemical imaging.

Acknowledgements We would like to thank the Electron Microscopy Center at ETH Zürich (EMEZ), and Frank Krumeich for performing the SEM analyses as well as Urs Stauffer and Laure Aeschimann (University of Neuchâtel) for supplying the $\mathrm{SiO}_{2}$ AFM probes. We would also like to acknowledge the Gebert-Rüf Foundation (grant nr. P085/03) and Deutsche Forschungsgemeinschaft (TS) for financial support of this project.

\section{References}

1. Stöckle RM, Suh YD, Deckert V, Zenobi R (2000) Chem Phys Lett 318:131-136
2. Anderson N, Anger P, Hartschuh A, Novotny L (2006) Nano Lett 6:744-749

3. Kikteva T, Star D, Zhao ZH, Baisley TL, Leach GW (1999) J Phys Chem B 103:1124-1133

4. Vannier C, Yeo BS, Melanson JE, Zenobi R (2006) Rev Sci Instrum 77:023104

5. Yeo BS, Zhang W, Vannier C, Zenobi R (2006) Appl Spectrosc 60:1142-1147

6. Hayazawa N, Inouye Y, Sekkat Z, Kawata S (2000) Opt Comm 183:333-336

7. Wang JJ, Saito Y, Batchelder DN, Kirkham J, Robinson C, Smith DA (2005) Appl Phys Lett 86:263111

8. Pan DH, Klymyshyn N, Hu DH, Lu HP (2006) Appl Phys Lett 88:093121

9. Rasmussen A, Deckert V (2006) J Raman Spectrosc 37:311-317

10. Neugebauer U, Rosch P, Schmitt M, Popp J, Julien C, Rasmussen A, Budich C, Deckert V (2006) Chemphyschem 7:1428-1430

11. Stöckle RM, Deckert V, Fokas C, Zenobi R (2000) Appl Spectrosc 54:1577-1583

12. Schatz GC, Van Duyne RP (2002) In: Chalmers JM, Griffiths PR (eds) Handbook of vibrational spectroscopy. Wiley, New York, pp 759-774

13. Campion A, Kambhampati P (1998) Chem Soc Rev 27:241-250

14. Kneipp K, Kneipp H, Itzkan I, Dasari RR, Feld MS (2002) J Phys-Condens Mat 14:R597-R624

15. Emory SR, Haskins WE, Nie SM (1998) J Am Chem Soc 120:8009-8010

16. Richards D, Milner RG, Huang F, Festy F (2003) J Raman Spectrosc 34:663-667

17. Hayazawa N, Inouye Y, Sekkat Z, Kawata S (2001) Chem Phys Lett 335:369-374

18. Mock JJ, Barbic M, Smith DR, Schultz DA, Schultz S (2002) J Chem Phys 116:6755-6759

19. Dieringer JA, McFarland AD, Shah NC, Stuart DA, Whitney AV, Yonzon CR, Young MA, Zhang XY, Van Duyne RP (2006) Faraday Discuss 132:9-26

20. Malinsky MD, Kelly KL, Schatz GC, Van Duyne RP (2001) J Phys Chem B 105:2343-2350

21. Xu G, Tazawa M, Jin P, Nakao S (2005) Appl Phys A 80:15351540

22. Xu G, Tazawa M, Jin P, Nakao S, Yoshimura K (2003) Appl Phys Lett 82:3811-3813

23. Philipp HR (1985) In: Palik ED (ed) Handbook of optical constants of solids, 1st edn. Academic, London, pp 765-769

24. Schneider S, Brehm G, Freunscht P (1995) Phys Status Solidi B 189:37-42

25. Pettinger B, Picardi G, Schuster R, Ertl G (2002) Single Mol 3:285-294

26. Saito Y, Wang JJ, Batchelder DN, Smith DA (2003) Langmuir 19:6857-6861

27. Norrod KL, Rowlen KL (1998) Anal Chem 70:4218-4221

28. Li M, Lin YC, Wu CC, Liu HS (2005) Nucl Acids Res 33: e184

29. Schlegel VL, Cotton TM (1991) Anal Chem 63:241-247

30. Heitmann W (1970) Thin Solid Films 5:61-67

31. Bradford AP, Hass G, McFarlan M, Ritter E (1965) Appl Opt 4:971-976

32. Cohen LF, Brown R, Milton MJT, Smith WE (eds) (2006) Faraday Discuss 132:1-340

33. Domke KF, Zhang D, Pettinger B (2006) J Am Chem Soc 128:14721-14727

34. Neacsu CC, Dreyer J, Behr N, Raschke MB (2006) Phys Rev B 73:193406

35. Hayazawa N, Inouye Y, Sekkat Z, Kawata S (2002) J Chem Phys 117:1296-1301 